\title{
Small Lymphocytic Lymphoma with Perifollicular, Marginal Zone, or Interfollicular Distribution
}

Dilip Gupta, M.D., Megan S. Lim, M.D., Ph.D., L. Jeffrey Medeiros, M.D., Kojo S.J. Elenitoba-Johnson, M.D.

From the Departments of Pathology, University of Utah Health Sciences Center, Salt Lake City, Utah (DG, KSJE-J), Sunnybrook and Women's College Health Science Centre, University of Toronto, Toronto, Canada (MSL), and University of Texas, M.D. Anderson Cancer Center, Houston, Texas (LM)

Chronic lymphocytic leukemia/small lymphocytic lymphoma (CLL/SLL) typically involves nodal or extranodal tissues as a diffuse proliferation with pseudofollicular growth centers obliterating normal architecture. We describe 16 cases of CLL/SLL in which the neoplasm was confined to the marginal zone, perifollicular, or interfollicular regions surrounding benign lymphoid follicles in either nodal or extranodal sites. Twelve of $12(100 \%)$ patients with adequate staging data had disseminated disease (Stage III or IV) at presentation. Eight of the 16 (50\%) patients had absolute peripheral lymphocytosis (range, 5 to $30 \times 10^{9} / \mathrm{L}$ ). Pseudofollicular growth centers were identified in 14 of 16 cases (87.5\%). Immunophenotypic studies revealed that the tumor cells were positive for $\operatorname{CD20}(16 / 16)$ and CD5 (11/11) in all cases examined. CD23 was positive in 12 of $14(86 \%)$ interpretable cases. IgM and IgD were positive in 13 of $14(93 \%)$ and 10 of 10 (100\%) interpretable cases, respectively. All cases were negative for $\operatorname{CD3}$ (16/16), CD45RO (16/16), CD10 (15/15), and cyclin D1 (15/15). We conclude that CLL/SLL can have unusual patterns of involvement, including marginal zone, perifollicular, and interfollicular patterns that can be difficult to recognize histologically. Thirteen of $16(81 \%)$ cases in this study were misinterpreted by the referring pathologists. Recognition of proliferation centers coupled with demonstration of a CD5+ CD23+ B-cell immunophenotype establishes the correct diagnosis of CLL/SLL.

Copyright (C) 2000 by The United States and Canadian Academy of Pathology, Inc.

VOL. 13, NO. 11, P. 1161, 2000 Printed in the U.S.A.

Date of acceptance: June 9, 2000.

Address reprint requests to: Kojo S. J. Elenitoba-Johnson, M.D. Division of Anatomic Pathology, University of Utah Health Sciences Center, 50 North Medical Drive, Salt Lake City UT 84132; e-mail: kojo.elenitobaj@path.med.utah.edu; fax: 801-585-3831.
KEY WORDS: CLL, Immunophenotype, Interfollicular, Marginal zone, Perifollicular, SLL.

Mod Pathol 2000;13(11):1161-1166

B-cell chronic lymphocytic leukemia/small lymphocytic lymphoma (CLL/SLL) is one of the most common lymphoproliferative disorders in western countries (1). Although most patients with CLL/SLL will ultimately demonstrate peripheral blood and bone marrow involvement (CLL), some cases exhibit exclusively nodal disease (SLL) at time of diagnosis (2). Both bone marrow or peripheral blood CLL and nodal-based SLL have identical morphologic and immunophenotypic characteristics. Thus, the International Lymphoma Study Group proposed the designation CLL/SLL to acknowledge that both CLL and SLL are currently considered to be different manifestations of the same disease entity (3).

Nodal involvement by CLL/SLL is most often characterized by complete effacement of nodal architecture by a diffuse proliferation of predominantly small lymphocytes characteristically interspersed with pseudofollicular growth centers (PFGC) or pseudofollicles, which are composed of increased numbers of prolymphocytes and paraimmunoblasts $(4-8)$. Less frequently (8 to $17 \%$ of cases), nodal involvement by CLL/SLL may be limited to the interfollicular areas (IF) $(2,6)$, leading to potential diagnostic confusion with benign interfollicular proliferations (5), and other neoplasms of small B-cells, such as marginal zone B-cell lymphoma (9), mantle cell lymphoma (10), and follicular lymphoma. The interfollicular pattern may also suggest peripheral T-cell lymphoma in some instances (5).

We describe 16 cases of CLL/SLL with marginal zone, perifollicular, and/or interfollicular patterns of involvement in nodal and extranodal sites. We emphasize the histopathologic and immunohistochemical features that are useful in recognition of this pattern of CLL/SLL, and review the features 
that assist in its distinction from other lymphoproliferative disorders.

\section{MATERIALS AND METHODS}

The files of the University of Utah Health Sciences Center, Salt Lake City, Utah, the University of Texas, M.D. Anderson Cancer Center, Houston, Texas, and the University of Toronto, Sunnybrook and Women's College Health Science Center, Toronto, Canada, during the period of 1994-1998 were searched for cases of CLL/SLL with interfollicular, perifollicular, and/or marginal zone patterns. Sixteen cases were identified. Clinical data (Table 1) were obtained from the referring physicians. Laboratory data, including the presence and pattern of bone marrow involvement, complete blood counts, serum immunoglobulin levels, and immunoelectrophoresis results were also obtained. Hematoxylin- and eosin- (H\&E) stained sections of B-5 and/or formalin-fixed, paraffin-embedded samples of nodal and extranodal tissue and bone marrow biopsy specimens were reviewed in all patients. Wright- and Giemsa-stained peripheral blood and/or bone marrow smears were available for review in nine cases. Touch imprints from lymph nodes were available for review in two cases.

Immunohistochemical studies were performed on all cases with either B-5 or formalin-fixed, paraffin-embedded tissue sections. Antigen retrieval was performed using microwave heat or enzymatic pretreatment (11). An avidin-biotinperoxidase complex method was performed using an automated immunostainer (Ventana Medical System, Tucson, AZ), and the following antibodies: immunoglobulin heavy $(\mu, \gamma, \alpha, \delta)$ and light $(\kappa, \lambda)$ chains, CD20, CD79a, BCL-2, CD3 (DAKO, Carpinteria, CA), CD5, CD10, (Novocastra, Newcastle-
upon-Tyne, UK), CD23 (Binding Site, San Diego, CA), CD43 (Becton Dickinson, San Jose, CA), CD45RO (Zymed, San Francisco, CA), and cyclin D1 (Neomarkers, Union City, CA).

The results of flow cytometric immunophenotypic analyses, all performed in the referring institutions, were available for four cases. In Case 1, lymph node, bone marrow, and peripheral blood specimens were analyzed. In Case 2 , lymph node and peripheral blood specimens were assessed. In the remaining two patients, lymph node biopsy specimens were studied.

\section{RESULTS}

\section{Clinical Data}

A summary of the clinical data is presented in Table 1 . There were eight men and eight women, with a mean age of 65 years (range, 38 to 88 years). Night sweats, fever, or weight loss ("B" symptoms) were present in two (12.5\%) cases. The most frequently biopsied sites were cervical (11 cases), inguinal (three cases), and axillary (one case) lymph nodes. In one case, CLL/SLL involved the colon in a resection specimen for poorly differentiated adenocarcinoma (Dukes stage A). Twelve of 12 (100\%) patients with adequate staging data had disseminated disease at presentation (Table 1). Spleen and bone marrow involvement was present in four and nine cases, respectively. CMV monospot test and Coomb's test were positive in one case each. Absolute lymphocytosis (more than $5 \times 10^{9} / \mathrm{L}$ ) indicating leukemic involvement (CLL) (12) was present in eight cases. The diagnoses rendered by the referring pathologists are summarized in Table 2.

Table 1. Clinical Data

\begin{tabular}{|c|c|c|c|c|c|c|c|c|c|c|c|c|}
\hline Case & Sex & $\begin{array}{l}\text { Age } \\
\text { (yr) }\end{array}$ & Biopsy Site & $\begin{array}{c}\text { WBC } \\
\left(/ \mathrm{mm}^{3}\right)\end{array}$ & $\begin{array}{l}\text { Pattern of } \\
\text { Involvement }\end{array}$ & BM & Liver & Spleen & Stage & $\mathrm{Rx}$ & Status & Other \\
\hline 1 & $\mathrm{~F}$ & 38 & Cervical & 8900 & IF & $\mathrm{Y}$ & $\mathrm{N}$ & $\mathrm{Y}$ & IV & CT & AWD & CMV, EBV \\
\hline 2 & $\mathrm{M}$ & 60 & Cervical & 7200 & IF & $\mathrm{N}$ & $\mathrm{N}$ & $\mathrm{Y}$ & III & CT & AWD & \\
\hline 3 & $\mathrm{~F}$ & 68 & Cervical & 8600 & IF & $\mathrm{Y}$ & $\mathrm{N}$ & $\mathrm{N}$ & IV & No & AWD & \\
\hline 4 & $\mathrm{~F}$ & 69 & Cervical & 8800 & IF & $\mathrm{N}$ & $\mathrm{N}$ & $\mathrm{N}$ & NA & RT & AWD & History of breast cancer \\
\hline 5 & $\mathrm{~F}$ & 88 & Axilla & 6200 & IF & $\mathrm{Y}$ & $\mathrm{N}$ & $\mathrm{N}$ & IV & No & AWD & \\
\hline 6 & $\mathrm{~F}$ & 68 & Inguinal & 5000 & IF & $\mathrm{N}$ & $\mathrm{N}$ & $\mathrm{N}$ & III & No & AWD & \\
\hline 7 & $\mathrm{~F}$ & 76 & Cervical & 9000 & IF & $\mathrm{N}$ & $\mathrm{N}$ & $\mathrm{N}$ & NA & No & AWD & \\
\hline 8 & $\mathrm{M}$ & 70 & Colon & 11200 & $\mathrm{PF} / \mathrm{IF}$ & $\mathrm{Y}$ & $\mathrm{N}$ & $\mathrm{N}$ & IV & No & AWD & Colonic adenoCA \\
\hline 9 & $\mathrm{M}$ & 45 & Inguinal & 3000 & $\mathrm{MZ} / \mathrm{D}^{*}$ & $\mathrm{Y}$ & $\mathrm{Y}$ & $\mathrm{Y}$ & IV & No & AWD & \\
\hline 10 & $\mathrm{M}$ & 74 & Cervical & 20000 & IF & $\mathrm{N}$ & $\mathrm{N}$ & $\mathrm{N}$ & NA & No & AWD & \\
\hline 11 & $\mathrm{M}$ & 66 & Cervical & 5700 & IF & $\mathrm{Y}$ & $\mathrm{N}$ & $\mathrm{N}$ & IV & CT & AWD & \\
\hline 12 & $\mathrm{~F}$ & 71 & Cervical & NA & MZ & $\mathrm{Y}$ & $\mathrm{N}$ & $\mathrm{N}$ & IV & CT & AWD & \\
\hline 13 & $\mathrm{M}$ & 67 & Cervical & 10000 & IF & $\mathrm{Y}$ & $\mathrm{Y}$ & $\mathrm{Y}$ & IV & CT & AWOD & \\
\hline 14 & $\mathrm{M}$ & 60 & Inguinal & 30000 & IF & $\mathrm{Y}$ & $\mathrm{N}$ & $\mathrm{N}$ & IV & CT & AWD & \\
\hline 15 & M & 66 & Cervical & 10000 & $\mathrm{MZ} / \mathrm{D}^{*}$ & $\mathrm{Y}$ & $\mathrm{Y}$ & $\mathrm{N}$ & $\mathrm{N}$ & IV & CT & AWD History of LBCL \\
\hline 16 & $\mathrm{~F}$ & 64 & Cervical & 10000 & IF & $\mathrm{N}$ & $\mathrm{N}$ & $\mathrm{N}$ & NA & No & AWD & \\
\hline
\end{tabular}

AWD, alive with disease; AWOD, alive without disease; DWD, dead with disease; BM, bone marrow; CMT, chemotherapy; Rx, treatment; IF, interfollicular; MZ, marginal zone pattern; PF, perifollicular; LBCL, large B-cell lymphoma; NA, not available; $\mathrm{D}^{*}$, diffuse involvement in a subsequently available lymph node specimen. 
Table 2. Diagnoses by the Referring Pathologists

\begin{tabular}{lc}
\hline \multicolumn{1}{c}{ Diagnosis } & Frequency \\
\hline CLL/SLL & 3 \\
Mantle cell lymphoma & 3 \\
Marginal zone B-cell lymphoma & 3 \\
Follicular lymphoma, grade I-II & 2 \\
Composite lymphoma (SLL/CLL with & 1 \\
$\quad$ follicular small cleaved lymphoma) & \\
Diffuse large cell B-cell lymphoma & 1 \\
Interfollicular Hodgkin's disease & 1 \\
Atypical interfollicular proliferation & 1 \\
Castleman's disease, hyaline-vascular type & 1 \\
\hline
\end{tabular}

CLL/SLL, chronic lymphocytic leukemia/small lymphocytic lymphoma.

\section{Histopathologic Findings}

In the lymph nodes, the distribution of CLL/SLL was predominantly interfollicular in 10 of $16(63 \%)$ cases (Fig. 1), interfollicular and focally diffuse in 3 of $16(19 \%)$ and predominantly marginal zone in 3 of 16 (18\%) cases (Fig. 2). The neoplasm was composed predominantly of small mature-appearing lymphoid cells with scant cytoplasm, round nuclei and clumped chromatin with "block-like" chroma-

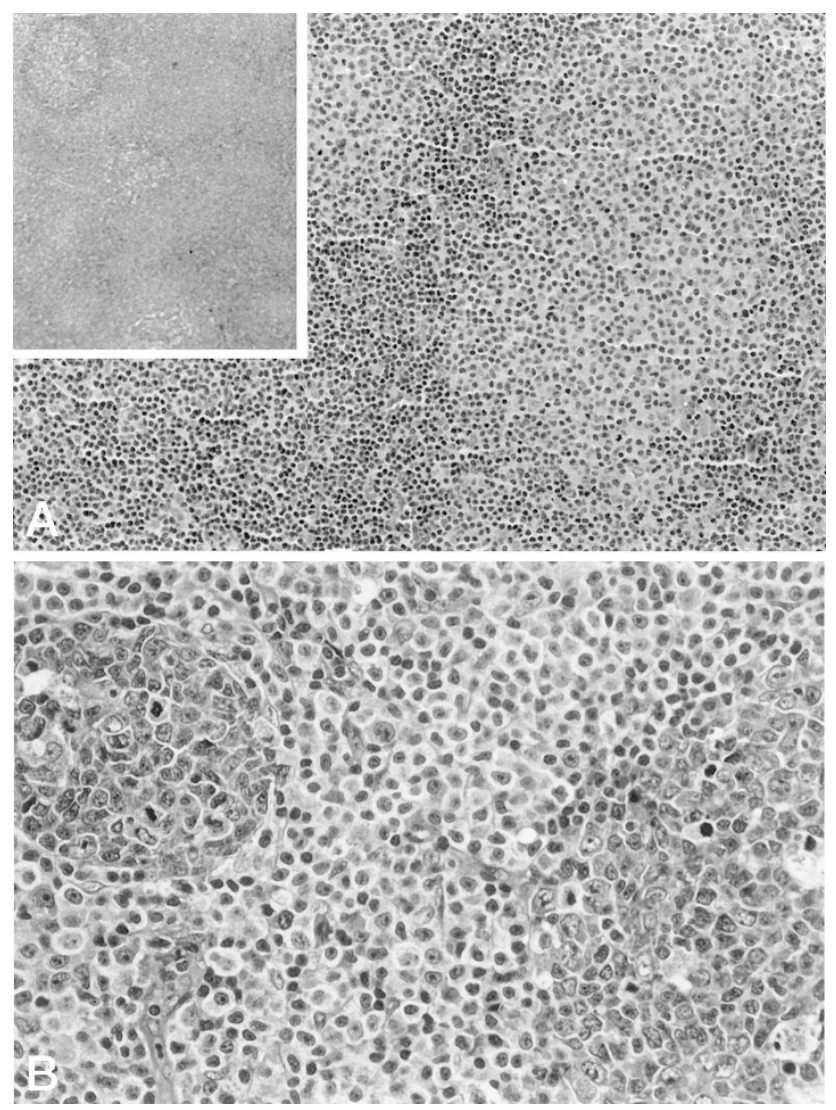

FIGURE 1. A, Medium-power photomicrograph showing pseudofollicular growth centers represented by pale staining nodules with prolymphocytes and paraimmunoblasts. Inset, low-power H\&E photomicrograph showing indistinct pale nodules (pseudofollicular growth centers) surrounding well-circumscribed reactive follicles (Case 2). B, H\&E stain showing a pseudofolicular growth centers (PFGC) between two reactive follicles. Majority of the cells in the interfollicular area are prolymphocytes and paraimmunoblasts with single central and prominent nucleoli and abundant pale cytoplasm.

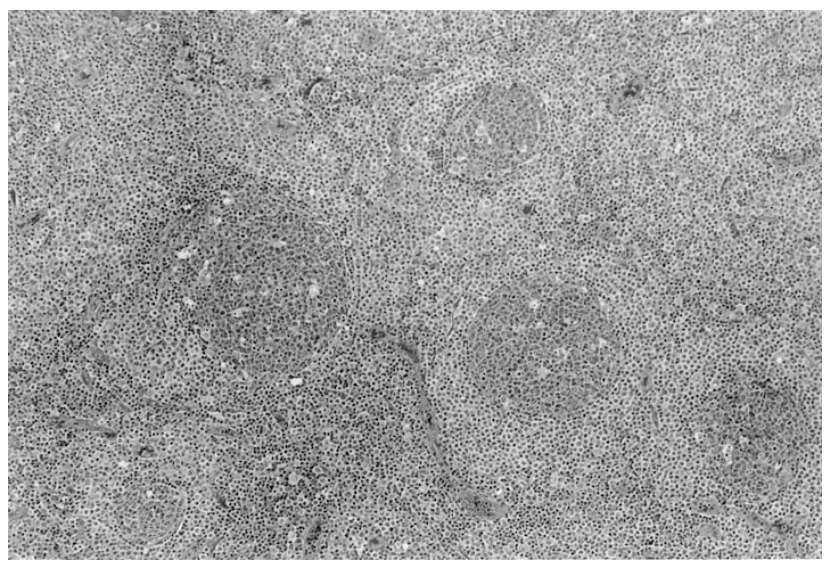

FIGURE 2. H\&E stain showing CLL/SLL with PFGC in a predominantly marginal zone distribution (Case 15). A subsequent biopsy specimen revealed diffuse involvement by classic CLL/SLL with the distinctive proliferation centers.

tin condensation. PFGCs were identified in 14 of 16 $(87.5 \%)$ cases. PFGCs were variable in size, and varied from 1 to 15 per low-power field (LPF), and contained variable numbers of small mature lymphocytes and larger cells with dispersed nuclear chromatin, single central nucleoli, and relatively abundant cytoplasm (prolymphocytes and paraimmunoblasts). Mitoses were infrequent (0 to $4 / 10$ HPF). Residual follicles with atrophic or large reactive germinal centers were present in all cases. Histologic examination of subsequently available material from two cases with marginal zone CLL/SLL (Cases 9 and 15) revealed diffuse nodal involvement by CLL with characteristic proliferation centers. The subcapsular sinuses were obliterated in 9 of 15 $(60 \%)$ or patent in 6 of $15(40 \%)$ cases. Extracapsular extension by the neoplasm was present in 6 of 15 (40\%) cases. There was evidence of bone marrow involvement in 8 of 16 cases. The bone marrow involvement was nodular in three of eight (37.5\%), interstitial in four of eight (50\%), and diffuse in one of eight (12.5\%) cases. Touch imprints obtained in both Cases 9 and 15 showed diffuse aggregates of small lymphocytes with mature clumped nuclei and scant cytoplasm. Occasional prolymphocytes and paraimmunoblasts with larger vesicular nuclei and prominent nucleoli and moderate cytoplasm were interspersed within the small lymphocytic infiltrate.

Two cases with nodal involvement by CLL/SLL in an interfollicular pattern also showed involvement of extranodal sites with a perifollicular or interfollicular growth pattern. In Case 8, involvement of the wall of the large intestine was observed as neoplastic small lymphocytes and PFGCs interspersed between well-developed lymphoid follicles with reactive germinal centers. In Case 13, PFGCs surrounded paratrabecular and nonparatrabecular reactive germinal centers in the bone marrow. 
Immunophenotypic Findings

\section{Paraffin section immunohistochemistry}

The neoplastic cells were positive for CD20 (dim as compared with the brighter residual follicles) (16/16) (Fig. 3), CD5 (11/11) (Fig. 4), CD23 (12/14) (Fig. 5), CD43 (16/16), CD79a (16/16), and BCL-2 (16/16). The neoplastic cells did not express CD3 (16/16), CD10 (15/15), CD45RO (16/16), or cyclin-D1 (15/15) in any of the cases examined. Proliferative activity, determined by MIB- 1 reactivity, was low (5 to $20 \%$ ) in the 11 cases tested, being mainly positive in the reactive germinal centers and focally positive in proliferation centers. Immunoglobulin (Ig) light chain restriction was demonstrated in 2 of 16 (12.5\%) cases by immunohistochemical studies using paraffin sections. In the remaining 14 cases, immunohistochemical studies were either technically unsatisfactory or inconclusive for light chain restriction. Immunoreactivity for IgM was demonstrated in 13 of 14 (93\%) cases, IgD in 10 of $10(100 \%)$ cases, and IgM and IgD were coexpressed in the 10 of $10(100 \%)$ cases in which both stains were interpretable.

\section{Flow cytometry}

Flow cytometric immunophenotypic analysis was performed in four cases using peripheral blood (two cases), bone marrow (one case), and lymph nodes (four cases). The results obtained by flow cytometry were identical to those obtained by paraffin immunohistochemistry for those antigens assessed by both methods. In all cases, the neoplastic cells displayed a B-cell phenotype positive for monotypic Ig light chain $(3 \kappa, 1 \lambda)$, CD19, CD20, CD5, and CD23.

\section{DISCUSSION}

CLL/SLL is a low grade B-cell neoplasm most often characterized by diffuse effacement of nodal

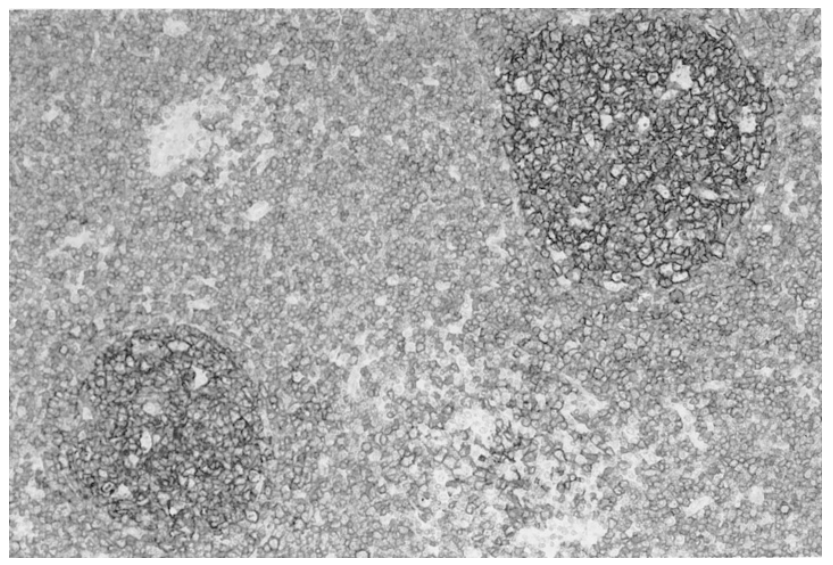

FIGURE 3. Immunohistochemical stain for CD20. Note "dim" reactivity in interfollicular CLL/SLL in comparison to the reactive germinal center with strong CD20 reactivity (Case 4).

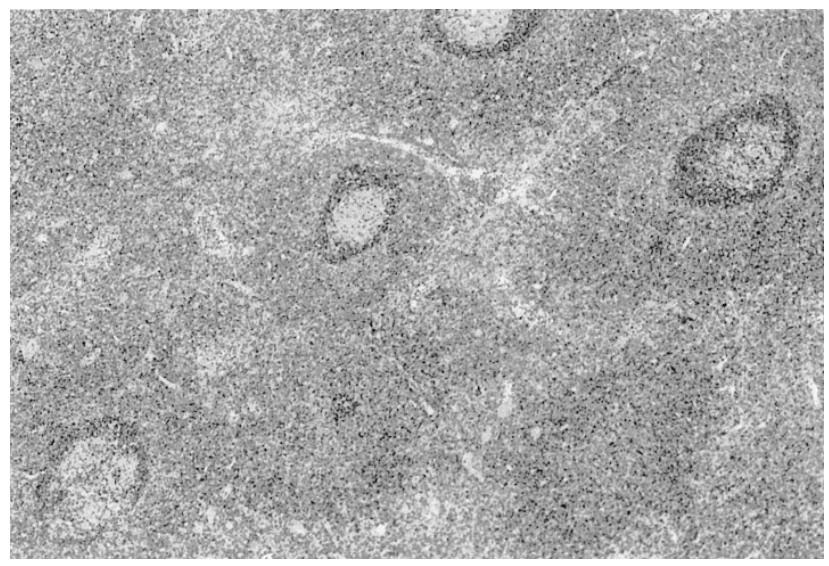

FIGURE 4. Immunohistochemical stain for CD5 showing coexpression by the neoplastic B-cells expanding the interfollicular zone (Case 1).

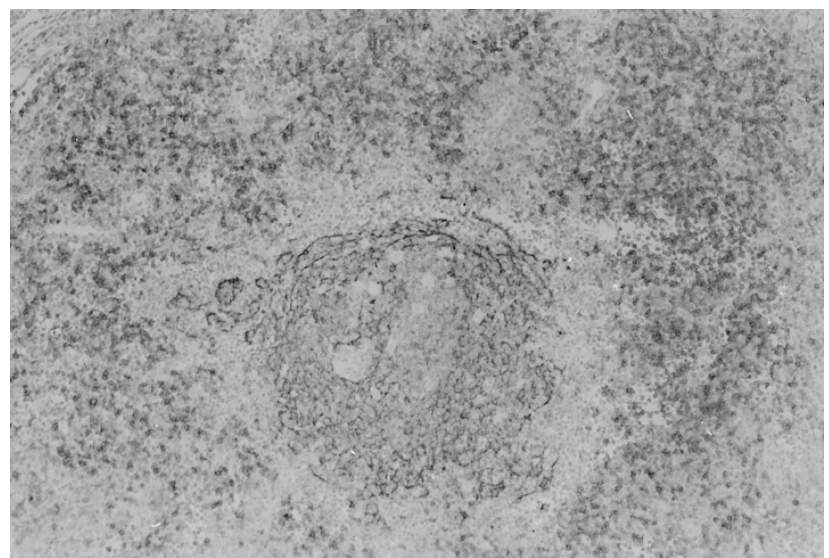

FIGURE 5. Immunohistochmical stain for CD23 showing reactivity within the follicular dendritic cell network and the interfollicular CLL/ SLL infiltrate (Case 1).

architecture by a proliferation of small mature appearing lymphocytes interspersed with PFGCs (4, 7). The PFGCs are pale at low power and impart a vaguely nodular appearance to the neoplasm. PFGCs are pathognomonic of CLL/SLL and contain increased numbers of prolymphocytes and paraimmunoblasts $(4,7)$. Though typically found in vaguely circumscribed aggregates, PFGCs may be inconspicuous when the prolymphocytes and paraimmunoblasts are loosely aggregated or scattered throughout the lymph node (13).

A small subset of CLL/SLL may show unusual patterns of involvement, and may selectively involve the interfollicular or marginal zones of lymph nodes $(2,6)$ or spleen (14). In these scenarios, CLL/ SLL may be histologically confused with other types of lymphoma such as mantle cell lymphoma, marginal zone B-cell lymphoma (9), follicular lymphoma, lymphoplasmacytic lymphoma, interfollicular Hodgkin's disease, and some peripheral T-cell lymphomas (8). CLL/SLL with interfollicular or marginal zone distribution also may be mistaken 
for non-neoplastic conditions such as reactive marginal zone or interfollicular hyperplasia. In our current study, MZ/IF- CLL/SLL was misinterpreted as another disorder in 13 of 16 cases including mantle cell lymphoma (three cases), marginal zone B-cell lymphoma (three cases), follicular lymphoma (two cases), atypical interfollicular hyperplasia (one case), and Castleman's disease, hyaline vascular type (one case).

Mantle cell lymphoma (MCL), in contrast to CLL/ SLL, is composed of a monotonous population of lymphoid cells, usually with irregular nuclear contours and scant cytoplasm. Importantly, larger transformed cells and proliferation centers are invariably absent (15). Recognition of proliferation centers is substantially enhanced by decreasing the amount of light that passes through the condenser at lower magnification $(5,8)$. This feature is particularly helpful in the recognition of the mantle zone pattern of MCL in which residual germinal centers may be present.

In contrast with CLL/SLL, marginal zone B-cell lymphoma (MZBCL) is composed of small cells with abundant clear cytoplasm, which is generally absent in CLL/SLL (9). Furthermore, convincing prolymphocytes and paraimmunoblasts are generally absent in MZBCL. Cytologic examination of the Wright-stained touch imprints may also be advantageous in the distinction of CLL/SLL from MZBCL, in that CLL/SLL is composed mostly of small mature appearing lymphocytes with round nuclei and scant basophilic cytoplasm. In contrast, MZBCL is composed of cells exhibiting round to irregular nuclei with abundant pale bubbly cytoplasm (16). In addition to the preceding morphologic features, nodal MZBCL may be immunophenotypically distinguished from CLL/SLL by its lack of expression of CD5, CD23, and $\operatorname{IgD}(3,17)$.

Misdiagnosis of CLL as follicular lymphoma (seen in two of our cases), or composite lymphoma (diagnosed as CLL/SLL with follicular lymphoma in one case), may be as a result of misinterpretation of the PFGCs as neoplastic follicles. In contrast to PFGC, the neoplastic follicles of follicular lymphoma are composed of atypical cells with cleaved nuclear contours, whereas the prolymphocytes and paraimmunoblasts of the proliferation centers characteristically show round nuclear contours and central nucleoli (3).

CLL/SLL frequently needs to be distinguished from lymphoplasmacytic (lymphoplasmacytoid) lymphoma/immunocytoma (LPL) (3). LPL is a tumor of small lymphoid cells with plasmacytoid or plasmacytic differentiation. The growth pattern is often interfollicular with sparing of the sinuses, and may bear a resemblance to IF-CLL/SLL. Nevertheless, LPL may be reliably distinguished from CLL/SLL by its lack of proliferation centers and the absence of CD5 and CD23 expression in the neoplastic LPL cells (3).

IF-CLL/SLL may also be mistaken on morphologic grounds for interfollicular Hodgkin's disease (HD) as occurred in one of our cases (Case 4). This occurs when the paraimmunoblasts of CLL/SLL are confused with mononuclear variants of ReedSternberg (RS) cells, so-called Hodgkin (H) cells. These RS/H cells of Hodgkin's disease may be immunophenotypically distinguished from the CD45, CD20, CD5, and CD23 positive cells of CLL/SLL by their characteristic CD45 negative, CD15, and CD30 positive phenotype (3).

In some cases of composite CLL/SLL and HD, an unwary observer may focus on the presence of the $\mathrm{RS} / \mathrm{H}$ cells and be oblivious of the underlying CLL/ SLL. The presence of a relatively monotonous background of small mature-appearing lymphocytes (in the absence of the other reactive cellular elements characteristic for HD) should alert the observer to the possibility of coexistent CLL/SLL (18). One of the cases (Case 5) was initially diagnosed by the referring pathologist as Castleman's disease, hyaline vascular type, because of atrophic germinal centers with prominent hyalinized central blood vessels and an interfollicular lymphoid infiltrate. However, diagnostic PFGCs were found within the interfollicular lymphoid infiltrate and permitted a correct diagnosis of CLL/SLL. In most cases, the PFGC of CLL/SLL can be distinguished from reactive germinal centers, as PFGCs are composed of round cells, lack mitotic figures, and tingible body macrophages, and are never circumscribed by mantle zones (4).

Immunophenotypic studies were very helpful in the recognition of MZ/IF-CLL/SLL. The tumor cells in MZ/IF-CLL characteristically showed dim reactivity by both flow cytometry and/or immunohistochemistry for CD20, which contrasted with the strong reactivity seen in the germinal center lymphocytes (Fig. 3). In all cases, the demonstration of coexpression of CD5, CD23, and CD43 (19) by the neoplastic cells in an interfollicular or marginal zone distribution supported the diagnosis of CLL/ SLL.

The explanation for the predominantly perifollicular, marginal zone, or interfollicular pattern in of CLL/SLL in lymph nodes is unclear. This microanatomic predilection may be explained, in part, by the putative origin of CLL/SLL from a CD5 + CD23+ $\mathrm{B}$-cell that is a recirculating pregerminal center B-cell, which resides or homes to the follicular mantle zone (20). Perhaps these cases represent partial or early involvement by neoplasm. However, the retention of these patterns in extranodal sites, and the presence of disseminated disease in most cases argue against this explanation. Alternatively, the neoplastic cells may secrete cytokines that in- 
duce germinal center formation, at least in extranodal sites. From a practical diagnostic standpoint, the recognition of these unusual patterns of CLL/ SLL is important. Once the possibility of CLL/SLL is considered, identification of the characteristic PFGCs or scattered prolymphocytes and/or paraimmunoblasts and the demonstration of the characteristic immunophenotypic profile will permit arrival at the correct diagnosis.

\section{REFERENCES}

1. Armitage JO, Weisenburger DD. New approach to classifying non-Hodgkin's lymphomas: clinical features of the major histologic subtypes. Non-Hodgkin's Lymphoma Classification Project. J Clin Oncol 1998;16:2780-95.

2. Ben-Ezra J, Burke JS, Swartz WG, Brownell MD, Brynes RK, Hill LR, et al. Small lymphocytic lymphoma: a clinicopathologic analysis of 268 cases. Blood 1989;73:579-87.

3. Harris N, Jaffe E, Stein H, Banks P, Chan J, Cleary M, et al. A revised European-American classification of lymphoid neoplasms: a proposal from the International Lymphoma Study Group. Blood 1994;84:1361-92.

4. Dick FR, Maca RD. The lymph node in chronic lymphocytic leukemia. Cancer 1978;41:283-92.

5. Ellison DJ, Nathwani BN, Cho SY, Martin SE. Interfollicular small lymphocytic lymphoma: the diagnostic significance of pseudofollicles. Hum Pathol 1989;20:1108-18.

6. Evans HL, Butler JJ, Youness EL. Malignant lymphoma, small lymphocytic type: a clinicopathologic study of 84 cases with suggested criteria for intermediate lymphocytic lymphoma. Cancer 1978;41:1440-55.

7. Lennert K. Malignant lymphomas other than Hodgkin's disease. New York: Springer-Verlag; 1978.

8. Nathwani B. Diagnostic significance of morphologic patterns in lymph node proliferations. In: Knowles D, editor. Neoplastic hematopathology. Baltimore: Williams \& Wilkins; 1992. p. 407-25.

9. Nguyen DT, Diamond LW, Schwonzen M, Bohlen H, Diehl V. Chronic lymphocytic leukemia with an interfollicular architecture: avoiding diagnostic confusion with monocytoid B-cell lymphoma. Leuk Lymphoma 1995;18:179-84.
10. Perry DA, Bast MA, Armitage JO, Weisenburger DD. Diffuse intermediate lymphocytic lymphoma. A clinicopathologic study and comparison with small lymphocytic lymphoma and diffuse small cleaved cell lymphoma. Cancer 1990;66: 1995-2000.

11. Bhan A. Immunoperoxidase. In: Colvin R, Bhan A, McCluskey R, editors. Diagnostic Immunopathology. 2nd ed. New York: Raven Press; 1995. p. 711-23.

12. Cheson BD, Bennett JM, Rai KR, Grever MR, Kay NE, Schiffer CA, et al. Guidelines for clinical protocols for chronic lymphocytic leukemia: recommendations of the National Cancer Institute-sponsored working group. Am J Hematol 1988; 29:152-63.

13. Pangalis GA, Nathwani BN, Rappaport H. Malignant lymphoma, well differentiated lymphocytic: its relationship with chronic lymphocytic leukemia and macroglobulinemia of Waldenstrom. Cancer 1977;39:999-1010.

14. Piris MA, Mollejo M, Campo E, Menarguez J, Flores T, Isaacson PG. A marginal zone pattern may be found in different varieties of non- Hodgkin's lymphoma: the morphology and immunohistology of splenic involvement by B-cell lymphomas simulating splenic marginal zone lymphoma. Histopathology 1998;33:230-9.

15. Banks PM, Chan J, Cleary ML, Delsol G, De Wolf-Peeters C, Gatter K, et al. Mantle cell lymphoma. A proposal for unification of morphologic, immunologic, and molecular data [see comments]. Am J Surg Pathol 1992;16:637-40.

16. Koo CH, Rappaport H, Sheibani K, Pangalis GA, Nathwani $\mathrm{BN}$, Winberg CD. Imprint cytology of non-Hodgkin's lymphomas based on a study of 212 immunologically characterized cases: correlation of touch imprints with tissue sections. Hum Pathol 1989;20(12 Suppl 1):1-137.

17. Campo E, Miquel R, Krenacs L, Sorbara L, Raffeld M, Jaffe ES. Primary nodal marginal zone lymphomas of splenic and MALT type. Am J Surg Pathol 1999;23:59-68.

18. Williams J, Schned A, Cotelingam JD, Jaffe ES. Chronic lymphocytic leukemia with coexistent Hodgkin's disease. Implications for the origin of the Reed-Sternberg cell. Am J Surg Pathol 1991;15:33-42.

19. Kumar S, Green GA, Teruya-Feldstein J, Raffeld M, Jaffe ES. Use of CD23 (BU38) on paraffin sections in the diagnosis of small lymphocytic lymphoma and mantle cell lymphoma. Mod Pathol 1996;9:925-9.

20. Kipps TJ. The CD5 B cell. Adv Immunol 1989;47:117-85. 